\title{
Determinantes de Longo Prazo do Produto e da Produtividade Total dos Fatores da Agropecuária Brasileira no Período 1974-2005
}

Henrique Brigatte ${ }^{1}$

Erly Cardoso Teixeira²

Resumo: O presente estudo desenvolve uma análise dosimpactos exercidos pelas mais relevantes variáveis no Produto Interno Bruto (PIB) e na Produtividade Total dos Fatores (PTF) da agricultura ao longo do período entre 1974 e 2005. São considerados como determinantes do crescimento econômico: investimentos em infraestrutura de transportes, de energia elétrica, de pesquisa, irrigação e armazenagem agrícolas, além dos montantes de crédito rural e da educação dos trabalhadores do setor agropecuário. A análise é feita por meio do uso de cointegração pelo método de Johansen, e os resultados indicam que educação e investimentos em infraestrutura exercem impactos positivos no longo prazo sobre o produto e a produtividade da agropecuária. Contrariamente, não são detectadas relações de cointegração entre crédito rural e o produto e a produtividade do setor.

Palavras-chave: Agropecuária brasileira, PTF, Cointegração.

Abstract: This study develops an analysis of the impacts of the most important variables in the Gross Domestic Product (GDP) and Total Factor Productivity (TFP) in agriculture in the period between 1974 and 2005. Investment in infrastructure for transport, energy, research, agricultural irrigation and storage and the amount of rural credit and education of workers in the agricultural sector are considered determinants of the economic growth. The analysis is done through the use of the Johansen cointegration method and the results suggest that education and investment in infrastructure have a positive impact on output and on agriculture yield in the long run. On the other hand,

\footnotetext{
1 Mestre em Economia Aplicada pela Universidade Federal de Viçosa. E-mail: hbrigatte@yahoo.com.br

2 Professor titular da Universidade Federal de Viçosa. E-mail: teixeira@ufv.br
} 
cointegration relationship between credit and production, and credit and agriculture yield were not detected.

Key-words: Brazilian agriculture, TFP, Cointegration.

Classificação JEL: O13, C22.

\section{Introdução}

É extensamente reconhecido que uma das condições fundamentais para a garantia da sustentabilidade no crescimento econômico de um país é a existência de infraestrutura adequada. Afinal, a infraestrutura disponível é responsável pela oferta dos recursos básicos que as empresas utilizam em seus processos produtivos. Além disso, o desenvolvimento das condições de infraestrutura deve ser acompanhado por avanços na concessão de crédito e aperfeiçoamentos constantes no capital humano.

$\mathrm{Na}$ agropecuária brasileira, é razoável pensar que aumentos nos investimentos em infraestrutura, nas possibilidades de financiamento e nas melhorias na educação dos seus trabalhadores podem levar a incrementos na produtividade, gerando, consequentemente, crescimentos no produto e na renda.

Num trabalho pioneiro explorando o tema, Aschauer (1989) evidencia a importância de investimentos públicos em infraestrutura, argumentando acerca do declínio da produtividade da economia norte-americana ocorrida na década de 1970. Ele relaciona tal fato às seguidas quedas nas taxas de investimento originado de capital público. A elasticidade encontrada para o produto, em relação ao volume desses investimentos públicos, foi de 0,39, indicando que, para cada $1 \%$ de aumento no estoque de capital público empregado para tais investimentos, ocorreria uma elevação de $0,39 \%$ na produção agregada.

Ferreira e Malliagros (1998), por exemplo, apresentam uma análise empírica do setor de infraestrutura brasileiro no período 1950-1995. Usando a metodologia econométrica de autorregressão vetorial (VAR), os autores concentram-se na estimação das elasticidades de longo prazo do produto e da PTF em relação ao capital e ao investimento em infraestrutura desagregados em cinco ramos - energia elétrica, telecomunicações, ferrovias, rodovias e portos. Seus resultados indicam a existência de forte relação entre infraestrutura, produto e produtividade no longo prazo, no Brasil.

Algumas pesquisas, como a conduzida por Gasques e Conceição (2000), fazem uso do índice de Tornqvist para a obtenção de séries de PTF da agricultura para os anos entre 1970 e 1995. Algebricamente, o índice de Tornqvist consiste 
numa aproximação discreta do índice contínuo de Divisia $^{3}$ e é calculado como a soma de razões logaritmizadas das quantidades de determinado produto e de determinado insumo em diferentes períodos de tempo, ponderadas, respectivamente, pelas participações do produto no valor total da produção e do insumo no custo total. Para que seja feita corretamente a construção da série da PTF por meio do índice de Tornqvist, é necessário que se possua disponibilidade de preços e quantidades referentes a todos os produtos e insumos considerados. Os resultados do trabalho de Gasques e Conceição (2000) indicam que a agricultura passou por ganhos expressivos de produtividade ao longo do intervalo de tempo analisado.

Um trabalho clássico que analisa a PTF por meio do índice de Divisia é o realizado por Jorgenson e Griliches (1967); as conclusões obtidas pelos autores são de que a PTF não mediria exatamente o efeito direto dado por uma nova tecnologia, mas as externalidades associadas ao processo de mudança tecnológica.

Morrison e Schwartz (1996), ao analisarem, para os Estados Unidos, a relação entre infraestrutura e produtividade em nível estadual, aplicando o referencial das teorias de produção por meio da abordagem das funções de custo das firmas, concluem que os investimentos em infraestrutura proporcionam significativos retornos e promovem aumentos na produtividade dos fatores das empresas. Ressaltam, todavia, que os benefícios sociais desses investimentos podem ou não ser positivos, dependendo dos custos sociais advindos da sua execução.

Zhang e Fan (2004), num estudo realizado para 290 distritos rurais da Índia, encontraram evidências de que investimentos públicos em infraestrutura geram efeitos positivos na PTF da agricultura indiana. Os valores das elasticidades da produção encontradas foram de 0,042 , com relação a investimentos públicos em rodovias, e de 0,081 para investimentos em irrigação.

Já Pires (2005) e Lucas (1993) procuram estabelecer uma relação entre crédito e desenvolvimento do capital humano e as condições para a consecução de crescimento econômico, respectivamente. $\mathrm{O}$ primeiro indica que há uma influência estatisticamente significativa do crédito no crescimento econômico, enquanto o segundo coloca o desenvolvimento do capital humano como condição essencial para a obtenção de um cenário favorável ao crescimento econômico e como principal fator explicativo das diferenças existentes entre os níveis de bem-estar social dos países.

Alguns trabalhos de Griliches (1963a, 1963b, 1964) procuram demonstrar a contribuição dada pela educação e pelos investimentos em pesquisa e

3 Um índice de Divisia consiste na soma ponderada de taxas de crescimento de componentes específicos, tais que as ponderações correspondem às participações desses componentes no valor total. 
desenvolvimento na produtividade da agricultura norte-americana. Os resultados indicam que tanto a educação quanto os investimentos em pesquisa e desenvolvimento têm reflexos positivos significativos no produto e na produtividade do setor, além de mostrarem que a taxa de retorno social transferida é expressiva.

Esse trabalho busca analisar as relações de longo prazo que o PIB e a PTF da agropecuária brasileira mantêm com os investimentos em infraestrutura realizados pelo governo federal no período entre 1974 e 2005. A opção em concentrar o foco neste período e nos investimentos federais deve-se à carência de dados estaduais.

Adicionalmente, mostrar-se-ão os efeitos exercidos por variáveis representativas de crédito rural e educação na produção e na produtividade da agropecuária brasileira. A pesquisa, desse modo, procura expor uma análise global acerca dos determinantes do crescimento de longo prazo da atividade agrícola do país.

\section{Referencial Teórico}

Os efeitos exercidos por aumentos na taxa de investimento sobre a atividade econômica de um país podem ser sintetizados pelo modelo de crescimento econômico de Solow. Ele fornece uma importante base para a compreensão das diferenças existentes entre os níveis de riqueza dos países, por meio de elementos que protagonizam papéis decisivos na determinação do investimento proporcional à produção bruta que determinada nação poderá efetivar.

Segundo Jones (2000), as conclusões alcançadas pelo modelo, em sua formulação original, indicam que os investimentos podem colaborar com o crescimento econômico. Demonstra-se que, no estado estacionário (em que o montante de capital por trabalhador permanece constante), um aumento na taxa de investimentos faz com que o nível de produção da economia cresça mais rapidamente, levando ao aumento do produto per capita e do volume de riqueza.

Posteriormente, a fim de tentar solucionar alguns problemas inerentes ao modelo de Solow, foram formuladas novas concepções teóricas, que colocaram o progresso tecnológico como elemento endógeno ao processo de crescimento.

A disponibilidade de créditoé outro fator que se configura como determinante do crescimento da economia. Para Lucas (1988), é especialmente importante o papel exercido pelos fatores financeiros na economia real, e a temática vem sendo cada vez mais discutida e abordada na literatura econômica.

O aperfeiçoamento do capital humano também repercute positivamente no crescimento da atividade produtiva. Jones (2000) estabelece uma versão simplificada do arcabouço teórico desenvolvido por Mankiw et al. (1992) para 
demonstrar que, no estado estacionário, a variável representativa do tempo que as pessoas despendem no acúmulo de conhecimento possui relação direta com o produto por trabalhador ao longo da trajetória de crescimento equilibrado; desse modo, é possível que incrementos na educação da população exerçam impactos positivos no nível de atividade econômica. Modelos endógenos de crescimento como o de Uzawa-Lucas também reforçam o caráter fundamental exercido pela educação no crescimento econômico.

\subsection{O resíduo de Solow}

O artigo de Solow (1957) representou o princípio de uma abordagem teórica mais profunda com respeito ao crescimento econômico. Em sua pesquisa, o autor propôs a realização de uma análise voltada à desagregação do crescimento econômico entre os já conhecidos fatores de produção dados pelo capital e pelo trabalho e um novo elemento passível de estudo, denominado por ele de mudança tecnológica, ou simplesmente tecnologia.

Este progresso tecnológico seria um elemento meramente residual, mas não menos importante, em termos quantitativos, para a explicação do crescimento. Esse resíduo concentraria toda a parcela do crescimento que não poderia ser explicada pela elevação dos fatores de produção usuais; tal componente passou a ser denominado de resíduo de Solow, que caracteriza o crescimento da Produtividade Total dos Fatores (PTF).

\section{Metodologia}

Este trabalho procura realizar uma investigação acerca dos efeitos causados no longo prazo pelos investimentos em infraestrutura, pelo crédito rural e pela educação dos trabalhadores agrícolas no PIB e na PTF da agropecuária brasileira.

No caso da análise voltada para a PTF, é preciso que se obtenha uma medida adequada para este índice de produtividade. No presente artigo, o procedimento é econométrico e tem como base o artigo de Solow (1957), no qual é desenvolvida uma espécie de contabilidade do crescimento em que se demonstra como poderia ser medido o progresso tecnológico (ou crescimento da PTF) a partir de uma função de produção do tipo Cobb-Douglas. Considera-se que as variações no produto que não são explicadas por variações no emprego dos fatores de produção capital e trabalho são explicadas pela PTF. Assim, a PTF pode ser dada como o resíduo da função de produção.

Portanto, analogamente a Mendes e Teixeira (2006), procedendo-se à estimação, por mínimos quadrados ordinários (MQO), da equação

$\ln Y_{t}=\beta_{1}+\beta_{2} \ln K_{t}+\beta_{3} \ln L_{t}+\varepsilon_{t}$ 
em que $Y_{t}$ representa o PIB agropecuário, $K_{t}$ o capital agropecuário e $L_{t}$, o trabalho no setor agropecuário medidos no tempo $t$, são obtidas as medidas das elasticidades do produto em relação ao capital e ao trabalho - dadas, respectivamente, por $\beta_{2}$ e $\beta_{3}$. Convém destacar que os resíduos dados por $\varepsilon_{\mathrm{t}}$ na equação (1) estão logaritmizados, uma vez que a especificação funcional adotada é log-log.

A estimação do estoque de capital do setor agropecuário foi feita com o uso do método do inventário permanente. A princípio, parte-se da lei de movimento do capital, dada por

$$
K_{t}=(1-\delta) K_{t-1}+I_{t}
$$

em que $\delta$ é a taxa de depreciação do capital agropecuário, e $I$ é o investimento realizado no período em estudo. Generalizada, a expressão assume a seguinte forma:

$$
K_{t}=(1-\delta)^{t} K_{0}+\sum_{j=0}^{t-1}(1-\delta)^{j} I_{t-j}
$$

A partir desta definição, e adotando-se o raciocínio proposto por Young (1995), alcança-se a equação utilizada para a determinação do estoque de capital inicial $K_{0}$ :

$$
K_{0}=\frac{I_{0}}{(\delta+g)}
$$

sendo $g$ a taxa média de crescimento do investimento no período anterior ao do estoque de capital inicial. Procedeu-se da mesma forma que Barros (1999) para a escolha da taxa de depreciação considerada neste trabalho, que foi de $6 \%$.

Assim, de posse das variáveis componentes da função de produção (1), tem-se que

$$
\hat{\varepsilon}_{t}=\ln \hat{Y}_{t}-\hat{\beta}_{1}+\hat{\beta}_{2} \ln K_{t}+\hat{\beta}_{3} \ln L_{t}
$$

representa o logaritmo da PTF. Para a obtenção da série da PTF, toma-se o antilogaritmo destes resíduos.

O procedimento para investigar se as variáveis consideradas neste trabalho mantêm relação de longo prazo com o PIB e a PTF da agropecuária encontra respaldo teórico em um conceito amplamente difundido em econometria, o de cointegração, estudado dentro dos conceitos de séries temporais.

A opção por modelos de cointegração ${ }^{4}$ se justifica nos casos em que as séries temporais analisadas são não estacionárias. Quando se diz que duas séries temporais são cointegradas, ou que uma delas cointegra com a outra,

4 Mais detalhes sobre o conceito de cointegração podem ser encontrados em Enders (1995). 
significa simplesmente que as mesmas guardam, entre si, uma relação de longo prazo, ou de equilíbrio. Algo extremamente importante, dado que a teoria econômica frequentemente é abordada em termos de equilíbrio (GUJARATI, 2006).

Neste trabalho, foram utilizados os procedimentos propostos por Johansen (1988) e Johansen e Juselius (1990), os quais consistem na estimação do(s) vetor(es) de cointegração a partir da construção de um modelo de vetor autorregressivo (VAR).

As relações de longo prazo mantidas pelo PIB e pela PTF da agropecuária com os determinantes considerados serão estabelecidas de acordo com as seguintes proposições formuladas por Ferreira e Malliagros (1998):

$$
\begin{aligned}
& \ln P I B_{t}=\phi \ln I_{t} \\
& \ln P T F_{t}=\eta \ln I_{t}
\end{aligned}
$$

em que $I_{t}$ representa um dos determinantes da análise. Lançou-se mão dessa formulação bivariada para o VAR (que fornecerá as conclusões relacionadas à existência ou não de cointegração entre o PIB e PTF da agropecuária e seus determinantes) pelo fato de as amostras colhidas terem sido de tamanho limitado. Dessa forma, de modo a realizar uma análise que não implicasse a perda de muitos graus de liberdade (a despeito da possibilidade de geração de vieses devido à omissão de variáveis relevantes no espaço de cointegração ${ }^{5}$ ), as relações foram tomadas por meio da construção de regressões simples.

Espera-se que os sinais das elasticidades representadas pelos coeficientes das regressões (6) e (7) sejam positivos; assim, a expectativa é de que $\phi>0$ e que $\eta>0$. Isso significaria que aumentos nos investimentos em infraestrutura, maiores montantes de crédito rural e aumento no nível de educação dos trabalhadores ocupados no setor agropecuário influenciariam positivamente o PIB e a PTF da agropecuária brasileira no longo prazo.

Os dados do PIB agropecuário anual (de 2000, em milhões de reais, deflacionados pelo deflator implícito do PIB), originados do IBGE (Instituto Brasileiro de Geografia e Estatística), foram obtidos no Instituto de Pesquisa Econômica Aplicada (Ipea).

Com relação à variável trabalho, que se refere ao número de trabalhadores formais empregados na agricultura, os dados foram colhidos por meio da série construída por Mendes e Teixeira (2006) para os anos de 1985 a 2005, e no IBGE para os anos de 1974 a 1984.

5 Tais vieses podem surgir a partir de possíveis subestimações ou superestimações dos coeficientes das variáveis que representam as elasticidades do produto e da PTF em relação aos determinantes mencionados. 
Os números do capital agropecuário [de 2000, em milhões de reais, deflacionados pelo Índice de Preços ao Consumidor (IPC) geral da Fundação Getúlio Vargas (FGV), cujos dados foram coletados no Ipea] foram estimados considerando-se o total de terras utilizadas para pastagens e para as principais culturas cultivadas no Brasil, além dos investimentos realizados pelo setor na compra de máquinas agrícolas automotrizes. Primeiramente, obtiveram-se, na FGV, os preços de venda de terras para pastagens e lavouras no período de 1977 a 2005 (encontrados em valores correntes, e posteriormente deflacionados pelo IPC geral para o ano-base de 2000). Em seguida, foi feita a multiplicação do total de áreas de pastagens (obtidas junto ao IBGE) e para o cultivo de algodão (arbóreo e herbáceo), arroz, cacau, café, cana, feijão, laranja, mandioca, milho, soja e trigo (medidos em hectares e obtidos na FGV) pelos valores dos preços coletados.

Em seguida, foi construída a série do estoque de capital de máquinas agrícolas automotrizes. Esse procedimento foi realizado por meio da aplicação dos métodos de Young (1995) e do inventário permanente aos dados de investimentos realizados na compra de máquinas agrícolas automotrizes, fornecidos pela Associação Nacional dos Fabricantes de Veículos Automotores (Anfavea). Tais valores, medidos em reais, de 2007, foram deflacionados para o ano-base de 2000 pelo IPC geral.

Para a obtenção do valor do estoque de capital inicial (ou seja, de 1977), foi aplicada a metodologia de Young (1995) aos dados de investimentos realizados na compra de máquinas agrícolas entre 1966 e 1976. Para alcançar os dados para os anos posteriores, empregou-se, recursivamente, a equação da lei de movimento do capital.

Assim, somaram-se os valores da utilização de terras para pastagens e lavouras à série do estoque de máquinas da agropecuária para obter uma aproximação da série do estoque de capital total do setor.

Desse modo, por meio das séries de PIB, trabalho e capital pertencentes ao setor agropecuário, foram obtidas as estimativas da PTF da agropecuária brasileira para o período de 1977 a 2005.

Quanto aos números de investimentos em infraestrutura no setor de transportes, as séries foram obtidas da base de dados de Ferreira e Malliagros (1998), para os anos de 1974 a 1995, e Ministério dos Transportes, de 1996 a 2005. Todos os valores foram deflacionados pelo IPC geral, para o ano-base de 2000 .

No setor de energia elétrica, a fonte de referência para a obtenção da proxy dos investimentos em eletricidade no meio rural, representada pelo consumo de energia elétrica do setor agropecuário, em GWh, foi a Empresa de Pesquisa Energética (EPE), do Ministério de Minas e Energia (MME).

Já os valores para a proxy de investimento em pesquisa agrícola foram coletados em Gasques et al. (2006). Os dados referem-se aos gastos executados 
pela Embrapa ao longo do período em estudo e foram deflacionados pelo IPC geral para o ano-base de 2000 (por escassez de dados, números de investimentos em pesquisa executados por institutos estaduais e por centros universitários importantes no estudo de questões ligadas à agricultura, que também constituem relevante parcela do investimento para o setor, não foram incluídos na série).

Os investimentos em técnicas de agricultura irrigada, representados pela proxy dada pelos hectares de terras irrigadas no Brasil, foram encontrados nos trabalhos de Lima et al. (2004) e Mendes e Teixeira (2006). Procedeu-se a interpolação, usando a taxa geométrica de crescimento, para os anos nos quais não havia disponibilidade de informações. Nas estimações, foram usados os dados de hectares de terras irrigadas proporcionais ao total de terras utilizadas no Brasil.

Os números da variável proxy considerada como investimentos em armazenagem agrícola, dada pela capacidade estática dos armazéns cadastrados na Conab (Companhia Nacional de Abastecimento), em mil toneladas, foram obtidos na Companhia. A insuficiência de dados fez com que a análise voltada para esta variável cobrisse somente o intervalo compreendido entre 1980 e 2005.

A variável representada pelo volume de crédito agrícola concedido ao setor agropecuário foi obtida por meio de dados coletados no Banco Central do Brasil (Bacen); os valores foram deflacionados pelo IPC geral para o ano-base de 2000. Esta variável também é uma proxy, pois nem todo o montante de crédito para a atividade agrícola é originado de instituições oficiais como o Bacen.

Por fim, os dados para a variável proxy educacional, dada pelo número médio de anos de estudo da população ocupada no setor agrícola, foram coletados de Freitas et al. (2007), e novamente foi utilizada interpolação pela taxa geométrica de crescimento para o preenchimento completo da série no período 1974-2005.

Todas as estimações econométricas foram realizadas com o uso do software estatístico Eviews 5.

\section{Resultados e discussões}

A série utilizada para o PIB agropecuário, em nível, é não estacionária a 5\% de significância estatística ${ }^{6}$; há a presença de somente uma raiz unitária, pois, tomando a primeira diferença da série, verificou-se que esta foi estacionária.

6 Conclusões obtidas pela execução do teste de raiz unitária ADF (Dickey-Fuller aumentado); optou-se pela omissão destes e outros resultados dos testes de raiz unitária por limitação de espaço. 
Dessa forma, a série é I(1), ou seja, integrada de ordem 1 . Todas as variáveis de infraestrutura, em nível, são também não estacionárias a pelo menos $10 \%$ de significância estatística; todas as séries possuem apenas uma raiz unitária, uma vez que, tomadas em suas primeiras diferenças, elas se tornaram estacionárias. Essas séries, portanto, são $I(1)$.

Dado que a série correspondente ao PIB agropecuário possui a mesma ordem de integração em relação a todas as variáveis de infraestrutura, pode-se admitir a possibilidade de que elas sejam cointegradas, ou seja, que elas possuam relação de longo prazo.

Assim, a estimação de modelos VAR bivariados foi realizada, conforme a especificação dada pela equação (6), para se estabelecer a relação de longo prazo baseada nesta mesma equação. A escolha quanto ao número adequado de defasagens foi feita de acordo com a minimização do Critério de Informação de Akaike (CIA), do Critério de Informação de Schwarz (CIS) e do Critério de Hannan-Quinn (HQ). Neste trabalho, os modelos foram construídos contendo entre uma e três defasagens.

Por meio dos procedimentos de Johansen (1988) e Johansen e Juselius (1990), mostram-se, na Tabela 1, os resultados dos testes de cointegração. 
Tabela 1. Resultados dos testes de cointegração entre as séries $L P I B_{t}$ e as séries de infraestrutura ${ }^{7}$.

\begin{tabular}{|c|c|c|c|c|c|c|c|}
\hline \multirow[t]{2}{*}{ Variáveis } & \multirow[t]{2}{*}{ Elasticidades } & \multicolumn{2}{|c|}{$\begin{array}{c}\text { Termos } \\
\text { deterministas } \\
\text { no vetor de } \\
\text { cointegração }\end{array}$} & \multirow[t]{2}{*}{$\begin{array}{l}\text { Hip. } \\
\text { nula }\end{array}$} & \multirow[t]{2}{*}{$\begin{array}{l}\text { Est. } \\
\text { traço }\end{array}$} & \multirow[t]{2}{*}{$\begin{array}{l}\text { Hip. } \\
\text { nula }\end{array}$} & \multirow[t]{2}{*}{$\begin{array}{l}\text { Est. Máx. } \\
\text { autovalor }\end{array}$} \\
\hline & & Tendência & Constante & & & & \\
\hline \multirow[t]{2}{*}{$\mathrm{LROD}_{\mathrm{t}}$} & 0,08 & - & 10,67 & $r=0$ & $19,08^{*}$ & $\mathrm{r}=0$ & $13,07^{\mathrm{ns}}$ \\
\hline & & & & $r \leq 1$ & $6,00^{\mathrm{ns}}$ & $\mathrm{r}=1$ & $6,00^{\text {ns }}$ \\
\hline \multirow[t]{2}{*}{ LFER $_{\mathrm{t}}$} & - & - & - & $r=0$ & $10,79^{\text {ns }}$ & $\mathrm{r}=0$ & $8,44^{\mathrm{ns}}$ \\
\hline & & & & $r \leq 1$ & $2,34^{\mathrm{ns}}$ & $\mathrm{r}=1$ & $2,34^{\text {ns }}$ \\
\hline \multirow[t]{2}{*}{$\mathrm{LPOR}_{\mathrm{t}}$} & - & - & - & $r=0$ & $12,47^{\mathrm{ns}}$ & $\mathrm{r}=0$ & $8,02^{\text {ns }}$ \\
\hline & & & & $r \leq 1$ & $4,45^{\mathrm{ns}}$ & $\mathrm{r}=1$ & $4,45^{\mathrm{ns}}$ \\
\hline \multirow[t]{2}{*}{$\mathrm{LEE}_{\mathrm{t}}$} & 0,65 & $-0,04$ & - & $r=0$ & $28,56^{* *}$ & $\mathrm{r}=0$ & $17,90^{\text {ns }}$ \\
\hline & & & & $r \leq 1$ & $10,66^{\mathrm{ns}}$ & $\mathrm{r}=1$ & $10,66^{\text {ns }}$ \\
\hline \multirow[t]{2}{*}{$\mathrm{LPES}_{\mathrm{t}}$} & 1,72 & - & - & $\mathrm{r}=0$ & $24,37^{* * *}$ & $\mathrm{r}=0$ & $24,36^{* * *}$ \\
\hline & & & & $\mathrm{r} \leq 1$ & $0,006^{\text {ns }}$ & $\mathrm{r}=1$ & $0,006^{\text {ns }}$ \\
\hline \multirow[t]{2}{*}{$\mathrm{LIR}_{\mathrm{t}}$} & - & - & - & $r=0$ & $15,05^{\mathrm{ns}}$ & $\mathrm{r}=0$ & $10,41^{\text {ns }}$ \\
\hline & - & & & $r \leq 1$ & $4,63^{\text {ns }}$ & $\mathrm{r}=1$ & $4,63^{\text {ns }}$ \\
\hline \multirow[t]{2}{*}{ LARM $_{\mathrm{t}}$} & 0,95 & - & - & $r=0$ & $19,45^{* * *}$ & $\mathrm{r}=0$ & $18,54^{* * *}$ \\
\hline & & & & $r \leq 1$ & $0,90^{\mathrm{ns}}$ & $\mathrm{r}=1$ & $0,90^{\mathrm{ns}}$ \\
\hline
\end{tabular}

ns: estatisticamente não significativo.

$*, * * \mathrm{e}^{* * *}$ : estatisticamente significativo a $10 \%, 5 \%$ e $1 \%$, respectivamente.

Nota: $L R O D_{t}, L F E R_{t}, L P O R_{t}, L E E_{t}, L P E S_{t}, L I R_{t}$ e $L A R M_{t}$ : investimentos em rodovias, ferrovias, portos, energia elétrica, pesquisa agrícola, irrigação e armazenagem em logaritmos, respectivamente.

Fonte: Resultados da pesquisa.

Pela Tabela 1, percebe-se que, para a variável de investimentos em rodovias, os resultados dos testes do traço e do máximo autovalor são contraditórios. No primeiro, é apontada a existência de um vetor de cointegração: a hipótese nula de que não existe um vetor de cointegração $(r=0)$ é rejeitada, enquanto a hipótese nula de que existe pelo menos um vetor de cointegração $(r \leq 1)$ não pode ser rejeitada a $10 \%$ de significância. No segundo teste, a hipótese nula de que não existe um vetor de cointegração $(\mathrm{r}=0)$ não pode ser rejeitada. Nesta e

7 Os sinais das elasticidades e dos termos deterministas são os das relações de longo prazo obtidas a partir dos vetores de cointegração, e não os dos elementos dos próprios vetores (que possuem sinais inversos). $\mathrm{O}$ mesmo vale para todos os testes de cointegração expostos na sequência. 
em outras situações em que isso ocorreu, foram considerados os resultados dos testes do traço. Por meio de simulações, Lütkepohl et al. (2001) demonstram que, em pequenas amostras (como as utilizadas no presente trabalho), o poder do teste do traço é superior ao do teste do máximo autovalor, o que pode levar à preferência pelo uso do primeiro em detrimento do último.

O sinal da elasticidade foi coerente com o esperado. Conforme o coeficiente obtido, para cada $1 \%$ de aumento nos investimentos em rodovias, o PIB agropecuário aumenta, em média, 0,08\%. O parâmetro, porém, não foi estatisticamente diferente de zero, como se pôde constatar por meio do valor da estatística $\mathrm{LR}^{8}$, o que limita tal interpretação.

Já as séries de investimentos em ferrovias e portos não cointegraram. A expressiva queda no volume de investimentos realizado pelo governo federal observada ao longo dos últimos anos para estes dois setores demonstra que a atividade agropecuária brasileira cresce, no longo prazo, independente dos avanços a cargo de investimentos públicos na malha ferroviária e na infraestrutura portuária. Esta situação, porém, pode não se sustentar, pois ambos os setores caracterizam infraestruturas sobejamente importantes para o escoamento da produção".

A série de energia elétrica cointegrou com o PIB agropecuário a 5\% de significância pelo teste do traço. O coeficiente da elasticidade, estatisticamente diferente de zero a $1 \%$ de significância pelo teste LR, demonstra que o PIB agropecuário responde, no longo prazo, com aumento de $0,65 \%$ a uma elevação média de $1 \%$ nos investimentos em eletricidade na área rural. Esta é uma relação plausível, dado que aumentos nos investimentos em eletricidade para o meio rural possibilitam a utilização de maior número de equipamentos mecanizados e mais modernos, o que conduz a um aumento na produção.

A variável de pesquisa na área agrícola cointegrou tanto no teste do traço quanto no teste do máximo autovalor a $1 \%$ de significância, e o parâmetro estimado mostra que aumentos de $1 \%$ neste tipo de infraestrutura repercutem em incremento médio de 1,72\% no nível de atividade do setor agropecuário. Este parâmetro também foi, a 1\% de significância, estatisticamente diferente de zero. Os ganhos proporcionados pela produtividade durante a década de 1980 para a produção agropecuária brasileira são evidências fortes de que a relação positiva entre produto e investimentos em pesquisa sustenta-se no longo prazo.

As variáveis de irrigação e de armazenagem denotam infraestruturas típicas da atividade agropecuária. A primeira não cointegrou com o PIB agropecuário nem mesmo a $10 \%$ de significância; esperava-se que estas variáveis fossem

8 Os resultados dos testes LR (razão de verossimilhança) de significância dos parâmetros do vetor de cointegração foram omitidos por limitação de espaço.

9 Apesar de reconhecer-se que a maioria da produção agropecuária escoada pelo território nacional utiliza transporte rodoviário. 
cointegradas. Assim, mesmo que tenha se percebido a evolução das áreas irrigadas, e que esses aumentos tenham ocorrido junto à evolução histórica do PIB agropecuário, não foi possível estabelecer, estatisticamente, uma relação de longo prazo entre irrigação e PIB agrícola.

Com relação à variável de armazenagem agrícola, observa-se que a elasticidade de longo prazo foi de $0,95 \%$, de modo que investimentos destinados à ampliação da capacidade estática dos armazéns podem gerar ganhos de longo prazo no volume de produção. O teste LR também confere respaldo estatístico para esse valor, com nível de significância de $5 \%$.

As séries de crédito rural e de educação também foram testadas quanto à sua estacionariedade. Ambas possuem uma raiz unitária, sendo, portanto, I(1). Como têm a mesma ordem de integração em relação à série do PIB agropecuário, é possível que estas sejam cointegradas. A Tabela 2 mostra os resultados.

Tabela 2. Resultados dos testes de cointegração entre as séries $L P I B_{t}$ e as séries de crédito rural e educação.

\begin{tabular}{|c|c|c|c|c|c|c|c|}
\hline \multirow[t]{2}{*}{ Variáveis } & \multirow[t]{2}{*}{ Elasticidades } & \multicolumn{2}{|c|}{$\begin{array}{l}\text { Termos determi- } \\
\text { nistas no vetor de } \\
\text { cointegração }\end{array}$} & \multirow[t]{2}{*}{$\begin{array}{l}\text { Hip. } \\
\text { Nula }\end{array}$} & \multirow[t]{2}{*}{ Est. traço } & \multirow[t]{2}{*}{$\begin{array}{l}\text { Hip. } \\
\text { Nula }\end{array}$} & \multirow[t]{2}{*}{$\begin{array}{l}\text { Est. máx. } \\
\text { autovalor }\end{array}$} \\
\hline & & Tendência & Constante & & & & \\
\hline \multirow[t]{2}{*}{ LCRED $_{\mathrm{t}}$} & 0,06 & - & - & $\mathrm{r}=0$ & $21,08^{* *}$ & $\mathrm{r}=0$ & $20,02^{* *}$ \\
\hline & & & & $r \leq 1$ & $1,06^{\mathrm{ns}}$ & $r=1$ & $1,06^{\mathrm{ns}}$ \\
\hline \multirow[t]{2}{*}{ LEDC $_{t}$} & 0,60 & - & 8,18 & $\mathrm{r}=0$ & $33,20^{* * *}$ & $\mathrm{r}=0$ & $24,84^{* * *}$ \\
\hline & & & & $\mathrm{r} \leq 1$ & $8,36^{\mathrm{ns}}$ & $r=1$ & $8,36^{\mathrm{ns}}$ \\
\hline
\end{tabular}

ns: estatisticamente não significativo.

** $\mathrm{e}^{* * *}$ : estatisticamente significativo a $5 \%$ e $1 \%$, respectivamente.

Nota: $L C R E D_{t}$, e $L E D C_{t}$ : crédito rural e anos de escolaridade dos trabalhadores agrícolas, respectivamente, em logaritmos.

Fonte: Resultados da pesquisa.

Nota-se, por meio da Tabela 2, que as duas séries cointegraram - crédito rural, a 5\% de significância, e educação, a 1\%. Com relação ao crédito rural, nota-se que a relação de cointegração foi detectada como significativa, de modo que, para cada $1 \%$ de elevação média no crédito rural, ocorre aumento de $0,06 \%$ no PIB agropecuário. Todavia, essa interpretação fica prejudicada pelo fato de o teste LR não garantir, estatisticamente, que tal parâmetro é diferente de zero. Sendo assim, não há garantia para a sustentação do argumento de que o crédito rural acompanhou, no longo prazo, o PIB agropecuário. Na verdade, isso não carece de plausibilidade: segundo Sant'Anna e Ferreira (2006), até meados da década de 1990, os recursos de crédito rural estiveram mais associados a investimentos 
especulativos (como aquisição de terras com o objetivo de proteção de renda, à época em que prevalecia um ambiente inflacionário, e posteriores operações de venda e de arrendamento a preços elevados) em detrimento dos produtivos, de modo que não era possível estabelecer uma associação entre montantes maiores de crédito com aumentos na produção agrícola. Essa situação somente se modificou a partir de 1996, quando o fim da inflação marcou a queda nos preços de venda e de arrendamento da terra, e a consequente interrupção de movimentos especulativos com as terras por meio do sistema de financiamento rural.

Quanto à educação dos trabalhadores ocupados nas zonas rurais brasileiras, percebe-se que o aumento médio de $1 \%$ nos anos de escolaridade deles produz elevação de 0,60\% no produto do setor agropecuário, no longo prazo. Tal parâmetro é válido a $1 \%$ de significância. A relação positiva era esperada, pois trabalhadores dotados de maior instrução deverão possuir maiores condições de especialização e maior nível de habilidade também nos campos, que utilizam cada vez mais bens de capital intensivos em tecnologia aplicados à produção.

\subsection{Relações de longo prazo da PTF da agropecuária brasileira}

A evolução da série da PTF da agropecuária brasileira foi obtida segundo os procedimentos explicados na seção 3 . A Figura 1 expõe a variação da série no período estudado.

Figura 1. Evolução da PTF da agropecuária brasileira: 1977/2005.

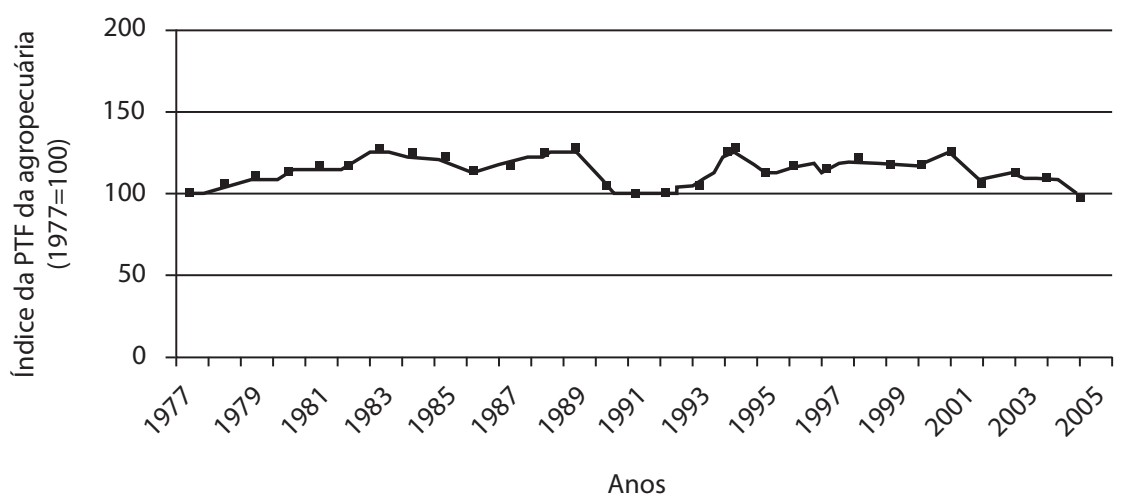

Fonte: Resultados da pesquisa.

O índice da PTF alcança seus maiores valores ao longo da década de 1980 e no início de 2000. Para os anos de 1980, a tendência da série atrela-se aos investimentos em pesquisa e em inovações na produção agropecuária, 
refletindo os gastos realizados nesta área, que alcançaram montantes expressivos principalmente entre o final dos anos 70 e o início da década de 1980. No início da década de 1990, observam-se quedas expressivas no índice de produtividade, possivelmente decorrentes dos diversos obstáculos conjunturais que pairaram sobre a economia brasileira à época.

De modo geral, pode-se dizer que houve ganhos na PTF do setor agropecuário brasileiro no intervalo em análise. A evolução média da PTF alcançou seu maior valor na década de 1980. Entre 1980 e 1989, por exemplo, a expansão alcançou $2,53 \%$, contra $0,47 \%$ do período inteiro. O valor da taxa média de crescimento da PTF encontrado para o intervalo como um todo é inferior se comparado aos obtidos por Gasques e Conceição (2000) e por Mendes e Teixeira (2006), de 2,33\% e de $1,03 \%$, respectivamente. Isto pode ter sido ocasionado pelos diferentes métodos de estimação empregados para a PTF em relação aos utilizados no presente estudo.

Ademais, o fato de ambos os trabalhos supracitados terem analisado períodos ligeiramente distintos dos apreciados nesta pesquisa pode ter contribuído para a disparidade verificada entre os números. A presente pesquisa, por exemplo, inclui na análise os anos de 2004 e 2005. Particularmente em 2005, ano em que ocorreu o agravamento da crise agropecuária iniciada em 2004, fatores como a política cambial, a queda dos preços internacionais da soja, milho e trigo e a estiagem que se abateu sobre grandes regiões produtoras do Brasil tornaram inevitáveis a quebra de safra e a redução da produtividade (FAEP, 2006).

O teste de raiz unitária para a série da PTF mostra que ela, em nível, é não estacionária a $1 \%$ de significância; possui, ainda, uma raiz unitária apenas, pois foi estacionária quando tomada em sua primeira diferença.

As séries dos determinantes da PTF foram todas não estacionárias a pelo menos 10\% de significância também para o período compreendido entre 1977 e 2005. Dessa forma, as séries são I(1), assim como a série da PTF. Isso indica que elas possuem a mesma ordem de integração, ou seja, é possível que a PTF da agropecuária mantenha relações de longo prazo com investimentos em infraestrutura, crédito e educação dos trabalhadores agrícolas. A Tabela 3 mostra os resultados da estimação da relação (7). 
Tabela 3. Resultados dos testes de cointegração entre as séries $L P T F_{t}$ e as séries de infraestrutura.

\begin{tabular}{|c|c|c|c|c|c|c|c|}
\hline \multirow[t]{2}{*}{ Variáveis } & \multirow[t]{2}{*}{ Elasticidades } & \multicolumn{2}{|c|}{$\begin{array}{l}\text { Termos determi- } \\
\text { nistas no vetor de } \\
\text { cointegração }\end{array}$} & \multirow[t]{2}{*}{$\begin{array}{l}\text { Hip. } \\
\text { nula }\end{array}$} & \multirow[t]{2}{*}{$\begin{array}{l}\text { Est. } \\
\text { traço }\end{array}$} & \multirow[t]{2}{*}{$\begin{array}{l}\text { Hip. } \\
\text { nula }\end{array}$} & \multirow[t]{2}{*}{$\begin{array}{l}\text { Est. máx. } \\
\text { autovalor }\end{array}$} \\
\hline & & Tendência & Constante & & & & \\
\hline \multirow[t]{2}{*}{$\mathrm{LROD}_{\mathrm{t}}$} & 0,55 & - & $-4,08$ & $r=0$ & $18,79^{*}$ & $\mathrm{r}=0$ & $12,35^{\text {ns }}$ \\
\hline & & & & $\mathrm{r} \leq 1$ & $6,43^{\text {ns }}$ & $r=1$ & $6,43^{\text {ns }}$ \\
\hline \multirow[t]{2}{*}{ LFER $_{t}$} & $-0,31$ & $-0,05$ & - & $\mathrm{r}=0$ & $24,98^{*}$ & $\mathrm{r}=0$ & $18,87^{*}$ \\
\hline & & & & $r \leq 1$ & $6,12^{\text {ns }}$ & $r=1$ & $6,12^{\text {ns }}$ \\
\hline \multirow[t]{2}{*}{$\mathrm{LPOR}_{\mathrm{t}}$} & 0,15 & - & $-0,77$ & $\mathrm{r}=0$ & $19,23^{*}$ & $\mathrm{r}=0$ & $14,36^{*}$ \\
\hline & & & & $\mathrm{r} \leq 1$ & $4,37^{\mathrm{ns}}$ & $\mathrm{r}=1$ & $4,37^{\mathrm{ns}}$ \\
\hline \multirow[t]{2}{*}{$\mathrm{LEE}_{\mathrm{t}}$} & 0,64 & - & $-6,48$ & $r=0$ & $20,50^{*}$ & $r=0$ & $12,88^{\text {ns }}$ \\
\hline & & & & $r \leq 1$ & $7,61^{\mathrm{ns}}$ & $r=1$ & $7,61^{\mathrm{ns}}$ \\
\hline \multirow[t]{2}{*}{ LPES $_{t}$} & 1,66 & $-0,01$ & - & $r=0$ & $28,18^{* *}$ & $\mathrm{r}=0$ & $16,79^{\text {ns }}$ \\
\hline & & & & $r \leq 1$ & $11,38^{\text {ns }}$ & $r=1$ & $11,38^{\text {ns }}$ \\
\hline \multirow[t]{2}{*}{$\mathrm{LIR}_{\mathrm{t}}$} & - & - & - & $\mathrm{r}=0$ & $16,81^{\mathrm{ns}}$ & $r=0$ & $10,32^{\text {ns }}$ \\
\hline & & & & $\mathrm{r} \leq 1$ & $6,49^{\text {ns }}$ & $r=1$ & $6,49^{\text {ns }}$ \\
\hline \multirow[t]{2}{*}{ LARM $_{t}$} & $-0,70$ & - & 8,13 & $r=0$ & $25,69^{* *}$ & $r=0$ & $20,57^{* *}$ \\
\hline & & & & $\mathrm{r} \leq 1$ & $5,12^{\text {ns }}$ & $r=1$ & $5,12^{\text {ns }}$ \\
\hline
\end{tabular}

ns: estatisticamente não significativo.

${ }^{*} \mathrm{e}^{* *}$ : estatisticamente significativo a $10 \%$ e $5 \%$, respectivamente.

Nota: $L R O D_{t}, L F E R_{t}, L P O R_{t}, L E E_{t}, L P E S_{t}, L I R_{t}$ e $L A R M_{t}$ : investimentos em rodovias, ferrovias, portos, energia elétrica, pesquisa agrícola, irrigação e armazenagem em logaritmos, respectivamente.

Fonte: Resultados da pesquisa.

Observa-se que aumentos médios de $1 \%$ nos investimentos em rodovias e portos levariam a elevações de $0,55 \%$ e $0,15 \%$ na PTF no longo prazo, a $5 \%$ de significância no teste do traço e a $10 \%$ em ambos os testes, respectivamente. Dessa vez, ao contrário do observado na análise para o PIB agropecuário, a significância estatística do parâmetro dos investimentos em rodovias é validada pelo teste LR, a 5\% de significância, o que indica que a produtividade agrícola responde positivamente aos investimentos federais em estradas. No tocante aos investimentos em infraestrutura portuária, percebe-se que, apesar da constatação de não ter havido relação de longo prazo destes com o PIB agropecuário, foram encontradas evidências de cointegração com relação à PTF (que também foram respaldadas pelos resultados do teste LR, a 10\% de significância). Deficiências na infraestrutura de portos podem se traduzir em maiores dificuldades nas importações de insumos importantes para a elevação da produtividade agrícola, 
como fertilizantes e adubos, e na exportação da produção. Assim, é essencial que investimentos sejam estimulados neste setor para a manutenção e evolução da produtividade da agropecuária brasileira.

No tocante a ferrovias e armazenagem, ambas tiveram confirmadas relações de cointegração com a PTF (a 10\% de significância, no caso de ferrovias, e a 5\% para armazenagem), porém com sinais contrários aos esperados. Os resultados do teste LR confirmam que esses coeficientes são válidos a $1 \%$ de significância; assim, é possível que se prossiga com a análise interpretativa das elasticidades. Com relação ao investimento federal em ferrovias (que já não havia cointegrado com relação ao PIB agropecuário, de acordo com resultados expostos na subseção 5.1.2), a ocorrência de um sinal incoerente pode encontrar alguma explicação no fato de vários ramais ferroviários terem sido desativados no período 1960- 1995 (FERREIRA e MALLIAGROS, 1998). Ora, isso talvez tenha impossibilitado a observação de uma relação direta no longo prazo entre investimentos públicos em ferrovias e a PTF da agropecuária.

Já para o caso de armazenagem, é provável que o fato de boa parte da infraestrutura de armazenagem estar localizada em áreas urbanas [conforme atestado por Mendes e Teixeira (2006)] possa ter algum poder explicativo para a obtenção de uma elasticidade com sinal inverso em relação àquele esperado (ainda que ele não tenha se verificado para o teste de cointegração do PIB agropecuário).

No caso da variável de investimentos em irrigação, é provável que a limitação provocada pela necessidade de interpolação da série de proporção de áreas irrigadas do total de terras utilizadas (já comentada anteriormente) possa ter colaborado para a inexistência de cointegração.

Os investimentos em energia elétrica voltados para as zonas rurais brasileiras tiveram relação de longo prazo positiva com a PTF. Concluiu-se que, para cada $1 \%$ de aumento médio nesses investimentos, haveria uma elevação de $0,64 \%$ na PTF (a 10\% de significância no teste do traço). O teste LR garante que tal parâmetro é, em termos estatísticos, diferente de zero a 10\% de significância. Isso demonstra que a expansão da energia elétrica nos campos contribui para a obtenção de ganhos de produtividade na agropecuária, por meio da maior possibilidade de uso de equipamentos mais modernos no processo produtivo.

Por sua vez, o valor da elasticidade da PTF em relação aos investimentos em pesquisa agrícola foi de 1,66. A significância estatística desse coeficiente foi confirmada pelo teste LR a 5\% de significância. Portanto, percebe-se que a pesquisa agrícola mantém relações de longo prazo tanto com o produto como também com a produtividade do setor agropecuário.

A repetição do processo utilizado para os testes anteriores - a correta especificação dos dois modelos VAR bivariados conforme (7) - antecedeu a estimação dos testes de cointegração da PTF com crédito rural e educação. Após 
a conclusão do procedimento, foram realizados os testes, cujos resultados estão expostos na Tabela 4.

Tabela 4. Resultados dos testes de cointegração entre as séries $L P T F_{t}$ e as séries de crédito rural e educação.

\begin{tabular}{|c|c|c|c|c|c|c|c|}
\hline \multirow{2}{*}{ Variáveis } & \multirow{2}{*}{ Elasticidades } & \multicolumn{2}{|c|}{$\begin{array}{c}\text { Termos deterministas no } \\
\text { vetor de cointegração }\end{array}$} & \multirow{2}{*}{$\begin{array}{l}\text { Hip. } \\
\text { Nula }\end{array}$} & \multirow{2}{*}{$\begin{array}{l}\text { Est. } \\
\text { Traço }\end{array}$} & \multirow{2}{*}{$\begin{array}{l}\text { Hip. } \\
\text { Nula }\end{array}$} & \multirow{2}{*}{$\begin{array}{l}\text { Est. máx. } \\
\text { autovalor }\end{array}$} \\
\hline & & Tendência & Constante & & & & \\
\hline \multirow[t]{2}{*}{ LCRED $_{\mathrm{t}}$} & - & - & - & $\mathrm{r}=0$ & $13,33^{\text {ns }}$ & $\mathrm{r}=0$ & $10,05^{\mathrm{ns}}$ \\
\hline & & & & $\mathrm{r} \leq 1$ & $3,27^{\mathrm{ns}}$ & $\mathrm{r}=1$ & $3,27^{\mathrm{ns}}$ \\
\hline \multirow[t]{2}{*}{ LEDC $_{t}$} & 1,09 & - & $-4,32$ & $\mathrm{r}=0$ & $20,56^{* *}$ & $\mathrm{r}=0$ & $16,58^{* *}$ \\
\hline & & & & $\mathrm{r} \leq 1$ & $3,98^{\mathrm{ns}}$ & $\mathrm{r}=1$ & $3,98^{\mathrm{ns}}$ \\
\hline
\end{tabular}

ns: estatisticamente não significativo.

**: estatisticamente significativo a $5 \%$.

Nota: $L C R E D_{t}$ e $L E D C_{t}$ : crédito rural e anos de escolaridade dos trabalhadores agrícolas, em logaritmos, respectivamente.

Fonte: Resultados da pesquisa.

Não é identificada qualquer relação de longo prazo entre a PTF e crédito rural (tanto pelo teste do traço quanto pelo do máximo autovalor, como mostra a Tabela 4). Assim, é mais prudente considerar que, no longo prazo, as melhorias ligadas à concessão de crédito rural não tenham exercido consideráveis efeitos sobre a PTF da agropecuária brasileira.

Finalmente, a educação dos trabalhadores empregados no setor analisado também possui relação de longo prazo positiva com a PTF; a 5\% de significância pelo teste do traço e pelo teste do máximo autovalor, verificou-se que uma elevação média de $1 \%$ nos anos de escolaridade desses trabalhadores promove aumento de $1,09 \%$ na produtividade dos fatores (elasticidade estatisticamente diferente de zero, a $1 \%$ de significância pelo teste LR).

\section{Conclusões}

Comprova-se que, no período de estudo 1974-2005, investimentos em energia elétrica, pesquisa agrícola e armazenagem aumentam o PIB agropecuário no longo prazo, sendo o efeito exercido pela pesquisa agrícola o maior entre os observados. Aumentos na educação dos trabalhadores agrícolas exercem impacto positivo no produto agropecuário; já os investimentos em rodovias, ferrovias, portos, irrigação, além de crédito rural, não mantêm relação de longo prazo com o PIB da agropecuária, no período estudado. 
Entre 1980 e 1989, a PTF teve taxa de crescimento de 2,53\%, contra 0,47\% do período inteiro. Com exceção dos investimentos em ferrovias e irrigação, todos os demais determinantes de infraestrutura têm relações de longo prazo com a PTF da agropecuária. Novamente, o impacto positivo exercido pela variável pesquisa agrícola é o maior, seguido pelos efeitos dos investimentos em energia elétrica, rodovias e portos. Educação também mantém relação positiva de cointegração com a PTF da agropecuária, ao contrário dos investimentos em ferrovias e armazenagem, cujas elasticidades apresentam sinal negativo. Crédito rural também não cointegra com a produtividade agrícola.

Conclui-se, assim, como fundamental que o governo reverta a morosidade na realização de investimentos em infraestrutura e implemente melhorias na educação rural, a fim de acelerar as possibilidades de expansão do produto e da produtividade da agropecuária brasileira.

\section{Referências Bibliográficas}

ASCHAUER, D. A. Is public expenditure productive? Journal of Monetary Economics, v. 23, no 2, p. 177-200, 1989.

BANCO CENTRAL DO BRASIL. Anuário Estatístico do Crédito Rural 2007. Brasília, 2007. Disponível em: http://www.bcb.gov.br/?RELRURAL2007. Acesso em: 20 out. 2008.

BARROS, A. L. M. Capital, produtividade e crescimento da agricultura: o Brasil de 1970 a 1995. 149 f. Tese (Doutorado em Economia Aplicada) - Escola Superior de Agricultura "Luiz de Queiroz", Universidade de São Paulo, Piracicaba, 1999.

COMPANHIA NACIONAL DE ABASTECIMENTO - CONAB. Disponível em: http://www.conab.gov.br/conabweb/download/armazenagem/serie_historica. xls. Acesso em: 15 out. 2008.

EMPRESA DE PESQUISA ENERGÉTICA - EPE. Consumo final e conservação de energia elétrica (1970-2005). Brasília: EPE, 2006.

ENDERS, W. Applied econometric time series. New York: John Wiley \& Sons, Inc., 1995.

FEDERAÇÃO DA AGRICULTURA DO ESTADO DO PARANÁ - FAEP. Queda nos preços das commodities afeta renda dos produtores rurais. Disponível em: http:// www2.faep.com.br/boletim/bi898/bi898pag04.htm. Acesso em: 28 out. 2009.

FERREIRA, P. C.; MALLIAGROS, T. G. Impactos produtivos da infra-estrutura no Brasil: 1950-1995. Pesquisa e Planejamento Econômico, Rio de Janeiro, v. 2, p. 315- 338, 1998. 
FUNDAÇÃO GETÚLIO VARGAS - FGV. Disponível em: http://fgvdados.fgv.br. Acesso em: 17 dez. 2008.

GASQUES, J. G.; CONCEIÇÃO, J. C. P. R. Produtividade total dos fatores na agricultura. Preços Agrícolas, Piracicaba, n. 165, p. 3-7, 2000.

GASQUES, J. G.; VILLA VERDE, C. M.; BASTOS, E. T. Gastos públicos em agricultura: retrospectiva e prioridades. Revista Economia, v. 7, no 4, p. 209-237, 2006.

GRILICHES, Z. Estimates of the aggregate agricultural production function from cross-sectional data. Journal of Farm Economics, v. 45, no 2, p. 419-428, 1963 a.

. The sources of measured productivity growth: U.S. agriculture, 1940-1960. Journal of Political Economy, v. 71, no 4, p. 331-346, 1963b.

. Research expenditures, education, and the aggregate agricultural production function. American Economic Review, v. 64, no 6, p. 961-974, 1964b.

GUJARATI, D. N. Econometria básica. Rio de Janeiro: Elsevier, 2006.

INSTITUTO BRASILEIRO DE GEOGRAFIA E ESTATÍSTICA - IBGE. Disponível em: http://www.ibge.gov.br. Acesso em: 11 set. 2008.

INSTITUTO DE PESQUISA ECONÔMICA APLICADA - IPEA. Disponível em: http://www.ipeadata.gov.br. Acesso em: 05 set. 2008.

JOHANSEN, S. Statistical analysis of cointegrating vectors. Journal of Economic Dynamics and Control, v. 12, p. 231-254, 1988.

JOHANSEN, S.; JUSELIUS, K. Maximum likelihood estimation and inference on cointegration - with applications to the demand for money. Oxford Bulletin of Economics and Statistics, v. 52, p. 169-210, 1990.

JONES, C.I. Introdução à teoria do crescimento econômico. Rio de Janeiro: Campus, 2000 .

JORGENSON, D. W.; GRILICHES, Z. The explanation of the productivity change. Review of Economic Studies, v. 34, no 3, p. 349-383, 1967.

LIMA, J. E. F. W.; FERREIRA, R. S. A.; CHRISTOFIDIS, D. O uso da irrigação no Brasil. Disponível em: http://www.cf.org.br/cf2004/irrigacao.doc. Acesso em: 08 set. 2008.

LUCAS, R. Making a miracle. Econometrica, v. 61, no 2, p. 251-272, 1993.

. On the mechanics of economic development. Journal of Monetary Economics, v. 22, p. 3-42, 1988. 
LÜTKEPOHL, H.; SAIKKONEN, P.; TRENKLER, C. Maximum eigenvalue versus trace tests for the cointegrating rank of a VAR process. The Econometrics Journal, v. 4, no 2, p. 287-310, 2001.

MANKIW, N. G.; ROMER, D.; WEIL, D. N. A contribution to the empirics of economic growth. The Quarterly Journal of Economics, v. 107, no 2, p. 407-437, 1992.

MENDES, S. M.; TEIXEIRA, E. C. Efeitos dos investimentos em infra-estrutura na produtividade total dos fatores na agricultura. In: TEIXEIRA, E. C.; BRAGA, M. J. (Eds.). Investimento e crescimento econômico no Brasil. Viçosa: UFV, DER, 2006.

MINISTÉRIO DOS TRANSPORTES. Disponível em: http://www.transportes. gov.br. Acesso em: 10 set. 2008.

MORRISON, C. J.; SCHWARTZ, A. State infrastructure and productive performance. The American Economic Review, v. 86, n. 5, p. 1095-1111, 1996.

PIRES, M. C. C. Crédito e crescimento econômico: evidências para os municípios brasileiros. VII Encontro de Economia da Região Sul - Anpec Sul, 2005.

SANT'ANNA, A. A.; FERREIRA, F. M. R. Crédito Rural: da especulação à produção. Visão do Desenvolvimento - BNDES, no 11, 2006.

SOLOW, R. M. Technical change and the aggregate production function. Review of Economics and Statistics, v. 39, no 8, p. 312-320, 1957.

YOUNG, A. The tiranny of numbers: confronting the statistical realities of the East Asian growth experience. The Quarterly Journal of Economics, v. 110, no 3, p. 641-680, 1995.

ZHANG, X.; FAN, S. How productive is infrastructure? A new approach and evidence from rural India. American Journal of Agricultural Economics, v. 86, no 2, p. 492-501, 2004. 
